\title{
Assessment of bleeding in eye surgery
}

\author{
R M TACKLEY, P J McKENZIE, A J BRON, AND T LEONARD* \\ From the Nuffield Department of Anaesthetics, John Radcliffe Hospital, Oxford
}

SUMmARY A new method for assessing microhaemorrhage in eye surgery was used in 16 patients for cataract extraction. It was shown that bleeding was greatest in the first five minutes and that it correlated with the surgeon's clinical estimate of blood loss. The method was used to assess the effect of two different general anaesthetic induction regimens on intraoperative bleeding.

In many types of eye surgery a small amount of bleeding makes a large difference to the operative field and ease of surgery. Etomidate is a relatively new intravenous general anaesthetic induction agent which has been shown to cause a fall in intraocular pressure.' It was noted that following the use of etomidate the eye appeared less injected, and surgeons spontaneously commented that the operating field was better than usual. A technique for measuring the bleeding in cataract surgery was therefore developed to compare the difference between the operative fields during cataract surgery after induction of anaesthesia with etomidate or thiopentone.

\section{Patients and methods}

Sixteen patients were studied who were undergoing elective intracapsular cataract extraction under general anaesthesia at the Oxford Eye Hospital. Their ages ranged from 58 to 92 , and their general medical fitness was based on the American Society of Anaesthesiologists' classification. Twelve were ASA 2, 3 were ASA 1, and 1 was ASA 3. The ASA gradings are: class 1 , a normal healthy patient; class 2 , a patient with mild systemic disease; class 3 , a patient with severe systemic disease which is not incapacitating; class 4 , a patient with an incapacitating systemic disease which is a constant threat to life; class 5 , a moribund patient not expected to survive longer than 24 hours with or without operation.

Two standard techniques of anaesthesia were used differing only in the choice of induction agent. Etomidate or thiopentone was chosen on a random basis, and only the anaesthetists knew which one was

${ }^{*}$ Health Education Unit, Oxford.

Correspondence to Dr R M Tackley, Department of Anaesthesia, Royal Corn:vall Hospital, Truro, Cornwall. used. Blood pressure was maintained within $\pm 20 \%$ of preinduction values, and the end-tidal carbon dioxide was maintained at $4 \%$ by intermittent positive pressure ventilation in all except four cases, when the monitor malfunctioned.

A standard ab externo technique of cataract extraction was used, with conjunctival flap, limbal groove, two preplaced and three postplaced $8 / 0$ virgin silk interrupted sutures, razor entry, and completion of the section with scissors. Chymotrypsin was used routinely, and the lens was cryoextracted.

The time from the start of induction to the first conjunctival incision was noted, and cellulose swabs were used frequently to absorb blood in the operative field. These were collected in batches covering each five-minute interval for the first 15 minutes, and the remaining swabs used were batched at the end of surgery. The surgeon was asked for his clinical assessment of bleeding on a scale of 0-10 (0 being equivalent to zero bleeding) at the time of each collection. Care was taken by the surgeons to collect all blood on the swabs.

Collected swabs were immediately placed into Drabkin's solution and the blood loss estimated in the laboratory at a later date. $5 \mathrm{ml}$ of the patient's venous blood was taken during the first five-minute swab collection period for haemoglobin estimation.

\section{ASSESSMENT OF BLOOD LOSS}

The swabs were collected directly into $20 \mathrm{ml}$ of Drabkin's solution in a universal tube. At the end of the operation the number of swabs in each tube was counted and extra prewetted swabs added as necessary, so that there was the same number in each tube. This was done in order to minimise effects due to absorption of haemoglobin on to the swabs. 
Haemoglobin was measured by conversion to cyanmethaemoglobin with Drabkin's reagent (Diagnostic Reagents Ltd, Thame). The absorption of the cyanmethaemoglobin at $540 \mathrm{~nm}$ was then compared with that of a standard solution. The precision of the method was $5 \%$ and the sensitivity was $1 \mu \mathrm{l}$. The haemoglobin content of the edetic acid (EDTA) sample of the patient's blood was measured in triplicate after dilution of $10 \mu \mathrm{l}$ in $4 \mathrm{ml}$ of Drabkin's solution. Calculation of the volume of blood taken up into swabs was as follows:

$$
\mathrm{V}=100 \times \mathrm{S} \times 1000 / 5 \times \mathrm{B} \mu \mathrm{l},
$$

where $\mathrm{V}$ is volume of blood, $\mathrm{S}$ is concentration of haemoglobin eluted into Drabkin's reagent, and B is the patient's peroperative haemoglobin concentration.

\section{Results}

Most of the blood losses occurred during the first fiveminute collection period, although there was a

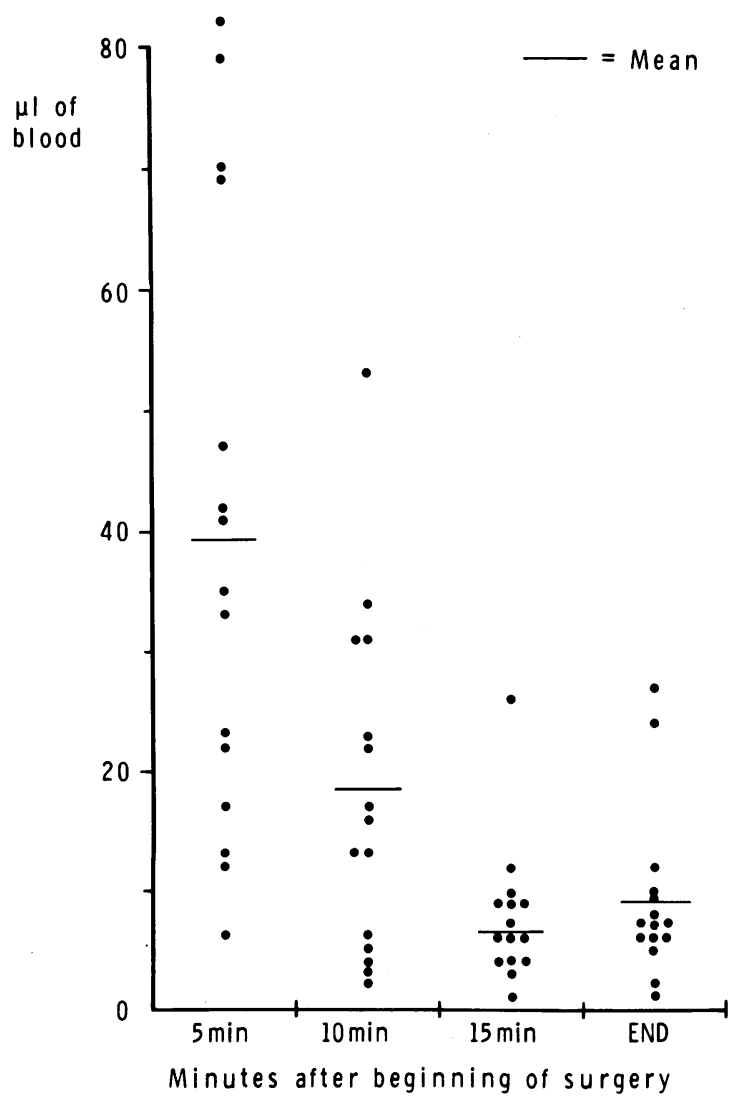

Fig. 1 Mean blood losses and the scatter of results during each collection period. marked variation in values (Fig. 1), which is in keeping with clinical experience.

The surgeon's clinical estimates of blood loss correlated well with the measured blood losses. The Spearman correlation coefficient was 0.711 $(p<0 \cdot 01)$. For this calculation the blood loss values were converted to a non-parametric scale.

There was no statistically significant relationship found between blood loss and the anaesthetic technique used. The mean interval from induction to operation was 14.6 min (range 9-23).

\section{Discussion}

It is generally held that the degree of congestion of the operative field and hence the likelihood of haemorrhage during surgery is influenced by anaesthetic agents and technique. Even small extravasations can obscure surgical detail during microsurgery, and therefore it was of interest to see whether such blood loss could be measured accurately over the operative course, and also to determine whether two different induction agents might have a different effect on blood loss during cataract surgery when a standard anaesthetic technique was used. Only one other study of this kind was found. ${ }^{2}$

Etomidate was chosen as a suitable induction agent for general anaesthesia because of its good cardiovascular stability, rapid metabolism, and ability to lower intraocular pressure.' The subjective observation by ophthalmic surgeons that its use was followed by less bleeding than with a standard thiopentone induction was not substantiated in the present study.

The method used here for measuring blood loss is accurate, sensitive (sensitivity $1 \mu \mathrm{l}$ compared with 5 $\mu \mathrm{l}$ in Clements's study ${ }^{2}$ ), and correlates well with clinical impression. Immediate immersion of swabs into Drabkin's solution allows a more complete elution of blood before it dries. The good correlation between measured blood loss and the surgeon's estimate suggests that the surgeon can make a reasonable assessment of blood loss during surgery.

In summary, it was not possible to demonstrate a difference in effect of two induction agents on microhaemorrhage into the operative field during cataract surgery. However, a technique has been described for the simple measurement of such haemorrhage which could be applied to the assessment of bleeding during other microsurgical procedures.

\section{References}

1 Oji EO, Holdcroft A. The ocular effects of etomidate. Anaesthesia 1979; 34: 245-9.

2 Clements DB. Ethamsalate in cataract surgery. Trans Ophthalmol Soc UK 1974; 94: 604-9. 